\title{
Dynamics of a single model neuron *
}

\author{
Frank Pasemann \\ Institut für Theoretische Physik \\ TU Clausthal, D-3392 Clausthal, FRG
}

\begin{abstract}
The parametrized dynamics of a standard nonlinear model neuron with self-interaction is discussed. For units with a self-excitatory connection a hysteresis effect is observed, and the underlying mechanism is identified as that of a cusp catastrophe. This is true for discrete as well as for continuous dynamics. For the discrete dynamics of self-inhibiting units there appear period-doubling bifurcations from stationary states to stable period-2 orbits.
\end{abstract}

*published in International Journal of Bifurcation and Chaos, 3, (1993), pp. 271-278. 


\section{Introduction}

With respect to information processing applications oscillatory dynamics so far is considered less important then convergent dynamics. In fact, most models of artificial neural networks, like e.g. Hopfield type networks [1], feedforward networks [2], and the class of network models satisfying the Cohen-Grossberg stability criterion [3], have convergent activation dynamics (see [4] for a review). This is a reasonable choice, since for applications like associative or content addressable memories, pattern classification, etc. one wants the input information to be represented by a stationary output of the network (see [5], [6] for anthology).

On the other hand, from nonlinear dynamics one knows that recurrent networks in general exhibit oscillatory or even chaotic dynamics. In fact, oscillatory dynamics in biological systems has been observed on various functional levels: For instance, it plays a role in central pattern generators, which drive rhythmic motor outputs [7]. In [8] and [9] it was suggested that odors are stored as limit cycles of the dynamics in the olfactory bulb, and that chaotic dynamics may play a role in olfactory information processing. Finally, cell assemblies in the visual cortex oscillating synchronously in response to external stimuli have been reported in [10], [11], [12].

First models simulating the olfactory dynamics [13], [14] or visual cortex dynamics [15], [16], [17], [18], [19], [20], [21] provide ideas, how the emergent properties of biological brains are related to complex dynamics, and how computational abilities may be generated by oscillatory networks. A collection of recent results can be found in [22].

The oscillatory dynamics of recurrent networks of reasonable size is in general difficult to describe, e. g. because mathematical tools for the analysis of periodic orbits in high dimensional phase spaces are still not sufficiently developed. But since network dynamics can be represented as a parametrized dynamical system on activation space [4], parameter studies of (discrete) neurodynamics can help to provide an understanding of the generic behavior of recurrent networks. This was demonstrated e. g. in [23], [24] and [25].

In this situation we suggest a modular approach to oscillatory neurodynamics, which will be described elsewhere. Nevertheless, as a very first step along this direction, this paper analyses the dynamics of single neuron. A simple additive nonlinear model neuron with self-interaction is chosen, and the corresponding dynamics is parametrized by the input $I$ and the self-connection $w$. The following questions are posed: What is the qualitative behavior in depence of the two parameters? Is there a difference between discrete and continuous dynamics?

Section 2 discusses the discrete dynamics, showing, that for self-excitatory units hysteresis effects and for self-inhibitory units stable oscillations can be observed for specific parameter domains. In Sec. 3 analysis of the continuous dynamics reveals hysteresis effects for the self-excitatory case but globally asymptotically stable [4] dynamics for the self-inhibitory unit. A summary of the results 
is given in Sec. 4.

\section{Discrete Dynamics}

We choose the standard additive nonlinear neuron model, i.e the activation $a_{i}$ of unit $i$ is given by the sum over the weighted outputs $o_{j}$ of units $j$ connected to unit $i$ plus a bias term $\theta_{i}$ :

$$
a_{i}:=\sum_{j=1}^{n} w_{i j} o_{j}+\theta_{i}
$$

The output $o_{i}$ of unit $i$ is given by a sigmoidal (S-shaped) transfer function $\sigma$, i.e. $\sigma$ is a bounded, monotone increasing function of the activation $a_{i}$. Here we choose

$$
o_{i}:=\sigma\left(a_{i}\right)
$$

with transfer function

$$
\sigma(a):=\frac{1}{1+e^{-a}}
$$

Grossberg [26] introduced sigmoidal transfer functions for contrast-enhancing the inputs and limiting the dynamic range of a unit. Since then this kind of nonlinear output has been used in a wide variety of neural network models, e.g. in [2] and [27].

We will consider the discrete dynamics of a single neuron with self-interaction $w$ (see Fig. 1) given by

$$
a(t+1):=w \cdot \sigma(a(t))+\theta \quad, \quad t \in \mathbf{N} .
$$

The parameter $\theta$ is interpreted here as the sum of the fixed bias $\theta_{0}$ and the variable total input $I$ of the unit. Thus the dynamics (4) is given by a two parameter

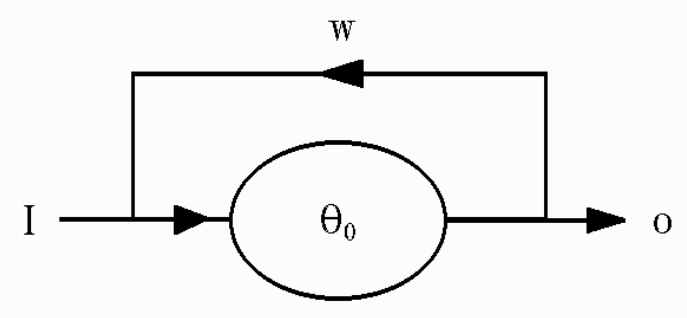

Figure 1: Model neuron with self-interaction.

family of maps $P: \mathbf{R}^{1} \rightarrow \mathbf{R}^{1}$

$$
a \mapsto P(a, \theta, w) \quad, \quad a \in \mathbf{R}^{1}, \quad(\theta, w) \in \mathbf{R}^{2} .
$$




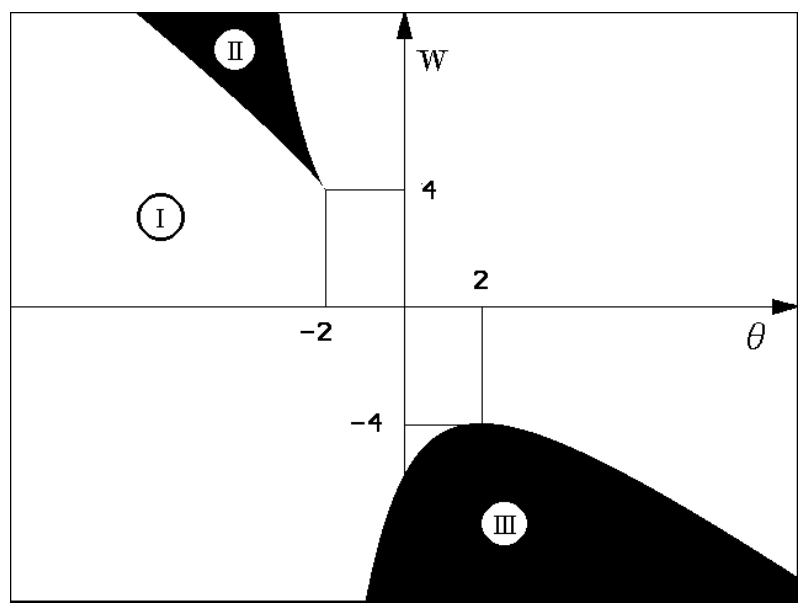

Figure 2: Parameter domains for I. one stable equilibrium, II. two stable, one unstable equilibrium, III. stable period-2 orbit and unstable equilibrium.

By simulating this dynamics we observe three different regions in the $(\theta, w)$ space (compare Fig. 2). In region I there exists a unique stable equilibrium for the system. To parameter values in region II there correspond three stationary states, one unstable and two stable, i.e. there are two coexisting fixed point attractors. In region III an unstable equilibrium and a stable period-2 orbit is observed.

The cubic nonlinearity of region II suggests that the mechanism of a cusp catastrophe [28], [29], [30], [31] may govern the dynamics in this self-excitatory $(w>0)$ domain. In fact, if for $w>4$ we pass forward and backward through the parameter domain $-10<\theta<0$, we observe the typical hysteresis effect for the output.

The existence of three stationary states $a^{*}$ for units with self-excitation $(w>$ 0 ) may be seen intuitively in Fig. 3a. As solutions of the fixed point equation

$$
a^{*}=P\left(a^{*}, \theta, w\right):=\theta+w \sigma\left(a^{*}\right),
$$

they are given by the intersection of the sigmoid (3) with the straight lines $f(a)=$ $(a-\theta) / w$.

The existence of a period-2 orbit for the self-inhibiting neuron $(w<0)$ can be visualized as in Fig. 3b. The orbit is given by the intersection of the function

$$
g(a):=\sigma(\theta+w \sigma(a))
$$

with the straight lines $f(a)=(a-\theta) / w$. This is derived from the period-2 orbit condition

$$
a=P^{2}(a):=\theta+w \sigma(\theta+w \sigma(a)) .
$$

Furthermore, the functions $\sigma, g$ and $f$ all intersect in one point corresponding to a stationary state $a^{*}$. 

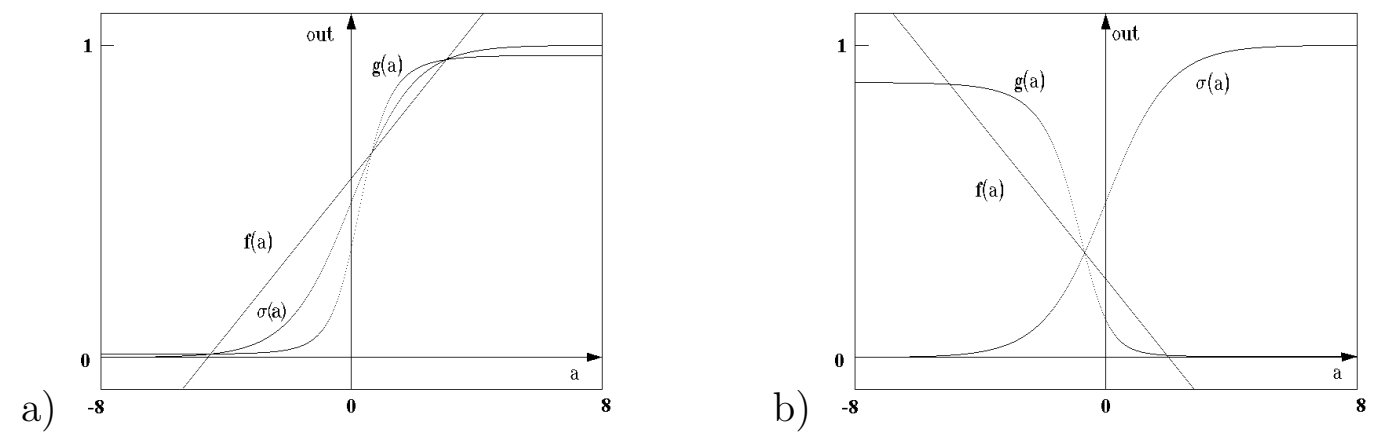

Figure 3: a) Three stationary states given by the intersection of the straight line $f(a)$ with the sigmoid $\sigma(a)$ b) Period-2 orbit given by the intersection of $f(a)$ with the function $g(a)$ of Eq. (7).

A stationary state $a^{*}$ is stable iff $\left|\frac{\partial}{\partial a} P\left(a^{*}\right)\right|<1$, i. e.

$$
\left|w \cdot \sigma^{\prime}\left(a^{*}\right)\right|<1
$$

Since $0<\sigma^{\prime}=\sigma(1-\sigma) \leq 1 / 4$, all stationary states $a^{*}$ are stable for $|w|<4$. Interesting dynamics therefore will be found in the domains $w \geq 4$ and $w \leq-4$ of $(\theta, w)$-space.

One may introduce as an additional control parameter the gain of the sigmoid by replacing the transfer function (3) by

$$
\sigma_{r}(a):=\sigma(r \cdot a) \quad, \quad r>0
$$

The stability condition (9) for a stationary state $a^{*}$ then reads

$$
\left|w \cdot r \cdot \sigma^{\prime}\left(a^{*}\right)\right|<1 \quad, \quad r>0 .
$$

For the moment we will fix the gain by setting $r=1$.

\subsection{Self-excitatory connections}

The boundary of region II $(w>0)$ is called the bifurcation set $B^{+}$. It is defined as the set of parameters values $(\theta, w) \in \mathbf{R}^{2}$ for which the stationary states $a^{*}$ given by equation (6) are non-hyperbolic with an eigenvalue of +1 , i.e. $\frac{\partial}{\partial a} P\left(a^{*}\right)=1$ or

$$
w \cdot \sigma^{\prime}\left(a^{*}\right)=1
$$

Especially, the fixed point $a^{*}=0$ is non-hyperbolic with eigenvalue +1 for the parameter values $\left(\theta_{c}, w_{c}\right)=(-2,4)$.

To verify the assumption that $B^{+}$corresponds to the bifurcation set of a cusp catastrophe [28], [29], [30], [31] we have to find a potential $V(a ; \theta, w)$ underlying 
the dynamics (4). Recall, if there exists such a potential $V$, the set $M \in \mathbf{R}^{1} \times \mathbf{R}^{2}$ of stationary states (here of the discrete dynamics) is given by

$$
M:=\left\{(a, \theta, w) \mid \frac{\partial V(a, \theta, w)}{\partial a}=0\right\},
$$

and the set $C \subset M$ of catastrophe points is given by

$$
C:=\left\{(a, \theta, w) \in M \mid \frac{\partial^{2} V(a, \theta, w)}{\partial a^{2}}=0\right\} .
$$

The projection of $C$ to the $(\theta, w)$-space is called the bifurcation set $B$ given by

$$
B:=\left\{(\theta, w) \in \mathbf{R}^{2} \mid \exists a \in \mathbf{R}^{1} \text { with }(a, \theta, w) \in M\right\} .
$$

Now, using the expansion of the sigmoid (3) around zero

$$
\sigma(a)=\frac{1}{2}+\frac{1}{4} a-\frac{1}{48} a^{3}+\frac{1}{480} a^{5}-\cdots,
$$

and using only terms up to order 3 , the condition for stationary states reads

$$
\frac{a-\theta}{w}=\sigma(a)=\frac{1}{2}+\frac{1}{4} a-\frac{1}{48} a^{3}
$$

Shifting the critical point $(\theta=-2, w=4)$ to the origin by replacing $\theta$ and $w$ by $\theta^{\prime}=\theta+2$ and $w^{\prime}=w-4$, the condition for the stationary states is given by

$$
a^{3}-12 \frac{w^{\prime}}{w^{\prime}+4} \cdot a-24 \frac{2 \theta^{\prime}+w^{\prime}}{w^{\prime}+4}=0
$$

The corresponding potential giving rise to this condition has the form

$$
V\left(a, \theta^{\prime}, w^{\prime}\right)=\frac{1}{4} a^{4}-6 \frac{w^{\prime}}{w^{\prime}+4} a^{2}-24 \frac{2 \theta^{\prime}+w^{\prime}}{w^{\prime}+4} a
$$

It can be transformed to the normal form of the universal unfolding [28] of the cusp catastrophe given by

$$
\tilde{V}(a, u, v)=\frac{1}{4} a^{4}+\frac{1}{2} u a^{2}+v a
$$

where

$$
u:=-12 \frac{w-4}{w} \quad, \quad v:=-24 \frac{2 \theta+w}{w} .
$$

The point in $M$ corresponding to $(u, v)=(0,0)$ or $\left(\theta_{c}, w_{c}\right):=(-2,4)$, respectively, is called the cusp catastrophe. This proves the following

Lemma 1 : The discrete dynamics (4) of a self-excitatory unit, i.e. $w>0$, is governed by a cusp catastrophe at $\left(\theta_{c}, w_{c}\right):=(-2,4)$. 
Near the cusp catastrophe the bifurcation set $B^{+}$is approximated by the equation

$$
(2 \theta+w)^{2}-\frac{4}{9} \cdot \frac{\left(w-w_{c}\right)^{3}}{w}=0
$$

For a fixed self-excitatory connection $\bar{w}>4$ the hysteresis effect appears over an $\theta$-interval with endpoints $\theta_{1}, \theta_{2}$ approximately given by

$$
\theta_{1,2}=-\frac{\bar{w}}{2} \mp \frac{\left(\bar{w}-w_{c}\right)}{3} \sqrt{\frac{\left(\bar{w}-w_{c}\right)}{\bar{w}}}
$$

Taking the gain parameter $\mathrm{r}$ into account, the stationary state $a^{*}$ is now given by the equation $a^{*}=\theta+w \cdot \sigma\left(r \cdot a^{*}\right)$. Using again the expansion (16) together with the stability condition (11) one gets for the cusp catastrophe

$$
\left(\theta_{c}, w_{c}\right)(r)=\left(-\frac{2}{r}, \frac{4}{r}\right) \quad, \quad r>0
$$

i.e. the cusp catastrophe moves along the straight line $2 \theta+w=0$ for $r \rightarrow \infty$ to the origin. The qualitative behavior remains the same as for $r=1$, but the bifurcation set $B^{+}$for $r \neq 1$ is shifted and slightly deformed: For a fixed selfconnection $\bar{w}>4 / r$ Eq. (23) for the endpoints of the hysteresis interval remains valid, with $w_{c}$ now given by Eq.(24).

At points $(\theta, w) \in B^{+}$in addition to the stationary state one stable and one unstable stationary state do appear (or disappear) (see Fig. 4a).

a)

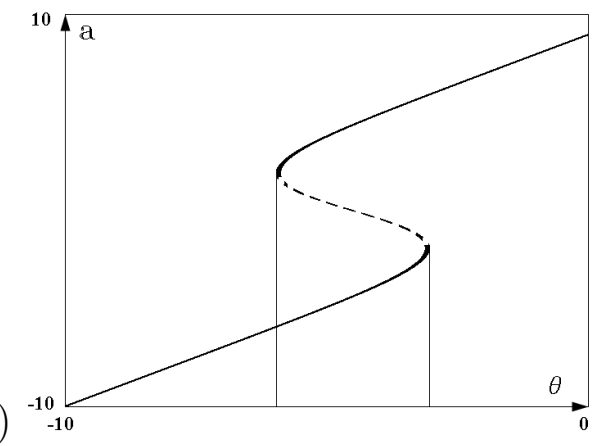

b)

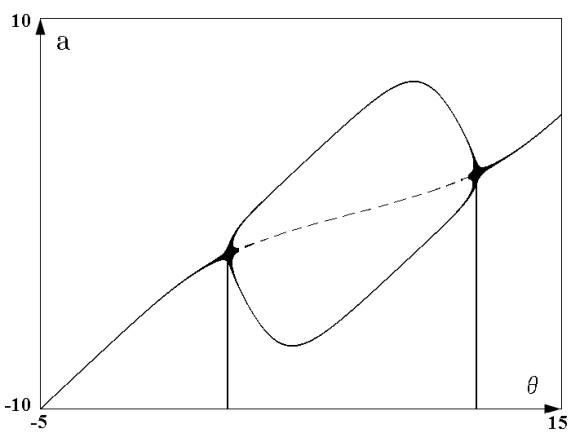

Figure 4: a) Stationary states for $w=8$, dashed line: unstable fixed point b) Period-doubling bifurcations for $w=-8$, dashed line: unstable fixed point.

\subsection{Self-inhibitory connections}

The boundary of region III in Fig. 2, called the bifurcation set $B^{-}$, is the set of parameter values $(\theta, w) \in \mathbf{R}^{2}$ for which the stationary states $a^{*}$ given by (6) are non-hyperbolic with an eigenvalue of -1 , i.e. $\frac{\partial}{\partial a} P\left(a^{*}\right)=-1$ or

$$
w \cdot \sigma^{\prime}\left(a^{*}\right)=-1
$$


Since $\sigma^{\prime}>0$ this can appear only for a self-inhibitory unit, i.e $w<0$. Especially, the fixed point $a^{*}=0$ is non-hyperbolic with eigenvalue -1 for the parameter values $\left(\theta_{c}, w_{c}\right)=(2,-4)$.

At this non-hyperbolic fixed point the following equations hold for the map $P^{2}$ :

$$
\begin{aligned}
& \frac{\partial}{\partial w} P^{2}=0 \quad, \quad \frac{\partial^{2}}{\partial a^{2}} P^{2}=0 \quad, \\
& \frac{\partial^{2}}{\partial a \partial w} P^{2} \neq 0 \quad, \quad \frac{\partial^{3}}{\partial a^{3}} P^{2} \neq 0 \quad \text {. }
\end{aligned}
$$

These equations provide sufficient conditions (compare e.g. [32], p. 373) for the system (4) to undergo a period-doubling bifurcation at the bifurcation point $\left(\theta_{c}, w_{c}\right)=(2,-4)$. This proves that at $B^{-}$we have bifurcations from a stable stationary state to a stable period-2 orbit (or the reverse) as shown in Fig. $4 \mathrm{~b}$.

Using again expansion (16) of $\sigma$ up to order 3 , the bifurcation set $B^{-}$is approximated near the singular point $\left(a, \theta_{c}, w_{c}\right):=(0,2,-4)$ by the following equation:

$$
(2 \theta+w)^{2}-\frac{4}{9} \cdot \frac{\left(w+2 w_{c}\right)^{2}\left(w-w_{c}\right)}{w}=0
$$

For a fixed self-inhibitory connection $\bar{w}<-4$ oscillations occur over an $\theta$ interval with endpoints $\theta_{1}, \theta_{2}$ approximately given by

$$
\theta_{1,2}=-\frac{\bar{w}}{2} \mp \frac{\left(\bar{w}+2 w_{c}\right)}{3} \sqrt{\frac{\left(\bar{w}-w_{c}\right)}{\bar{w}}}
$$

Again, the introduction of a gain parameter $r \neq 1$ will not change the qualitative behavior of the unit, however the bifurcation set $B^{-}$is shifted and slightly deformed. The critical point

$$
\left(\theta_{c}, w_{c}\right)(r)=\left(\frac{2}{r}, \frac{-4}{r}\right) \quad, \quad r>0
$$

is once more shifted to the origin with increasing $r$, and the oscillatory interval for fixed $\bar{w}<-4 / r$ is approximated by Eq. (29) with $w_{c}$ now given by (30).

\section{Continuous Dynamics}

We will now compare the results obtained for the discrete dynamics with the additive continuous dynamics of a single unit given by

$$
\dot{a}=-\gamma a+\theta+w \sigma(a), \quad \gamma>0,
$$

where $\gamma$ represents the decay rate. Analogously to the discrete case, we consider the right hand side as a two parameter vector field $f(a, \theta, w)$; i.e. $\gamma$ will be 
considered as a constant. For $\gamma=1$ a discretized version of this equation is given by

$$
a(t+\Delta t)=(1-\Delta t) a(t)+\Delta t(\theta+w \sigma(a)) \quad, \quad t \in \mathbf{R},
$$

which corresponds to the discussed dynamics (4) if $\Delta t=1$.

From the point of bifurcation theory, the continuous dynamics is less rich than the discrete one, since oscillations cannot occur for one dimensional systems. We will show that the continuous dynamics of the self-excitatory neuron is again controlled by a cusp catastrophe potential.

The fixed points $a_{0}$ of the dynamics (31) are solutions of the equation

$$
-\gamma a_{0}+\theta+w \sigma\left(a_{0}\right)=0 .
$$

A bifurcation will appear [32], if the fixed point $a_{0}$ becomes non-hyperbolic, i.e. if $D_{a} f\left(\left(a_{0}, \theta_{0}, w_{0}\right)=0\right.$ or

$$
-\gamma+w \cdot \sigma^{\prime}\left(a_{0}\right)=0
$$

Since $\gamma>0$ and $\sigma^{\prime}(a)>0$ for all $a$, this can only happen iff $w>0$. The fixed point $a_{0}=0$ is non-hyperbolic for the critical parameter values

$$
\left(\theta_{c}, w_{c}\right)=(-2 \gamma, 4 \gamma)
$$

Thus for $w<4 \gamma$ the system will have a unique stable equilibrium.

Since every one dimensional dynamics can be written as a gradient dynamics, we get

$$
f(a, \theta, w)=-\frac{\partial V(a, \theta, w)}{\partial a}
$$

Using again the expansion (16) of $\sigma$ around $a=0$ we have

$$
f(a, \theta, w)=\frac{1}{2}(2 \theta+w)+\frac{1}{4}(w-4 \gamma) a-\frac{1}{48} w a^{3} \cdots,
$$

and the condition (33) for stationary states reads

$$
a^{3}-12 \frac{(w-4 \gamma)}{w} a-24 \frac{(2 \theta+w)}{w}=0
$$

Shifting the critical point $\left(\theta_{c}, w_{c}\right)$ to the origin by introducing $\theta^{\prime}=\theta+2 \gamma$ and $w^{\prime}=w-4 \gamma$ this condition transforms to

$$
a^{3}-12 \frac{w^{\prime}}{w^{\prime}+4 \gamma} \cdot a-24 \frac{2 \theta^{\prime}+w^{\prime}}{w^{\prime}+4 \gamma}=0
$$

and the desired potential $V$ has the form

$$
V\left(a, \theta^{\prime}, w^{\prime}\right)=\frac{1}{4} a^{4}-6 \frac{w^{\prime}}{w^{\prime}+4 \gamma} a^{2}-24 \frac{2 \theta^{\prime}+w^{\prime}}{w^{\prime}+4 \gamma} a .
$$

By a diffeomorphism $V$ can be transformed again into the normal form (20) of the universal unfolding of the cusp catastrophe. The cusp catastrophe is now lying at $\left(a, \theta_{c}, w_{c}\right)=(0,-2 \gamma, 4 \gamma)$. This proves 
Lemma 2 : The continuous dynamics (31) of a self-excitatory unit, i.e. $w>0$, is governed by a cusp catastrophe at $\left(\theta_{c}, w_{c}\right)=(-2 \gamma, 4 \gamma)$.

We observe, that for $\gamma=1$ the last two equations are identical with (18) and (19), respectively. For self-excitatory connections $w>0$ the bifurcation set $B \subset \mathbf{R}^{2}$ for the continuous dynamics (31) with $\gamma=1$ is identical with the bifurcation set $B^{+}$of the discrete dynamics.

The bifurcation set near the cusp catastrophe is again approximately described by Eq. (22), where $\left(\theta_{c}, w_{c}\right)$ is now given by (35). The effect of a decreasing decay rate $\gamma \rightarrow 0$ on $B$ is the same as an increasing gain parameter $r \rightarrow \infty$ on $B^{+}$: the cusp is shifted to the origin. Furthermore for $\gamma=1 / r$ the bifurcation sets $B$ and $B^{+}$are identical.

\section{Discussion}

A single unit without self-interaction behaves as a simple non-linear input/output map, i.e. $o=\sigma\left(\theta_{0}+I\right)$. Interesting effects show up if self-connections $w$ are introduced.

For a self-excitatory connection $w>0$ discrete and continuous dynamics show qualitatively the same behavior. It is characterized (compare region II of Fig. 2) by the following

Statement 1 : The discrete and continuous dynamics of a single self-excitatory unit is governed by the potential of a cusp catastrophe $\left(\theta_{c}, w_{c}\right)$. For $0<w<w_{c}$ the unit has a unique fixed point attractor for all input values $I \in \mathbf{R}^{1}$. For $w>w_{c}$ and a zero bias term $\theta_{0}$ there exists an interval of negative inputs for which the unit has three stationary states, two stable and one unstable. Varying the inputs over this intervall results in a hysteresis effect.

Using the gain $r$ of the sigmoid as an additional control parameter of the discrete dynamics will not change its qualitative behavior. Variation of $r$ will move (24) the cusp catastrophe along the straight line $2 \theta+w=0$, such that the critical value $w_{c}$ approaches zero with increasing $r$. For a fixed connection $\bar{w}>w_{c}$ the hysteresis interval is growing with increasing $r$.

For the continuous dynamics, setting $r=1$, the same holds true (35) for a decreasing decay rate $\gamma$.

Functionally, hysteresis phenomena where discussed as models for short-term memory of neural systems for instance in [33], [34] and [35]. For single neurons hysteresis effects where described e.g. in [36].

The cusp catastrophe already played a central role in Zeeman's model of nerve cell dynamics [37]. His nerve membrane equations provided a simpler alternative to the well-known Hodgkin-Huxley equations [38]. 
For units with self-inhibitory connections $w<0$ discrete and continuous dynamics are quite different. While the continuous dynamics is globally asymptotically stable, i.e. has a unique fixed point attractor for all inputs $I \in \mathbf{R}^{1}$, the discrete dynamics (compare region III of Fig. 2) shows stable oscillatory behavior:

Statement 2 : For the discrete dynamics of a single self-inhibitory unit there exists a critical value $w_{c}<0$ with the following property: For $w_{c}<w<0$ the unit has a unique fixed point attractor for all input values $I \in \mathbf{R}^{1}$. For $w<w_{c}$ there exits an interval of input values for which the unit has a period-2 orbit attractor. At the border of these intervals there appears a period-doubling bifurcation. Thus self-inhibitory units can serve as oscillators.

With increasing gain parameter $r$ of the sigmoid the critical value $w_{c}$ shifts toward the origin $(30)$. The critical point $\left(\theta_{c}, w_{c}\right)$ for the self-inhibitory unit is the point reflection of the cusp catastrophe of the self-excitatory unit.

Finally, it should be emphasized that the qualitative results, i.e. hysteresis effect and period-2 orbits, are independent of the particular choice (3) of the sigmoidal transfer function. Recall that a sigmoid is defined as a bounded monotone increasing function. Different sigmoids will have different critical parameter values $\left(\theta_{c}, w_{c}\right)$. For example, with Eqs. (6), (9) and $r=1$ the critical values for a "balanced" sigmoid

$$
\sigma(a)=-1+\frac{2}{1+e^{-a}}
$$

are given by $\left(\theta_{c}, w_{c}\right)=(0, \pm 2)$, and for tanh as transfer function we get $\left(\theta_{c}, w_{c}\right)=$ $(0, \pm 1)$. Like the last two examples, antisymmetric sigmoids, i. e. $\sigma(-a)=$ $-\sigma(a)$, will generate bifurcation sets $B^{+}$and $B^{-}$symmetric around $\theta=0$.

Using the definitions in [4], the difference between discrete and continuous dynamics can be summerized in

Statement 3 : The continuous additive dynamics (31) of a single unit with selfconnection is convergent for all parameter values. The discrete dynamics (4) of a self-inhibitory unit displays stable oscillations for a distinct parameter domain.

Although the single units have an easy to control dynamics, already recurrent neuro-modules with only two units show an extremely rich behavior. In a forthcoming paperit is demonstrated, that the discrete dynamics of a 2-module with units having no self-connections display hysteresis effects, bifurcation to coexistent period- 2 orbits and two fixed points, and bifurcations to period- 4 orbits (compare also [25]). Allowing self-connections, simulations display various period-n orbits, with n odd or even, quasiperiodic orbits and even chaotic attractors. Various bifurcation scenarios like period-doubling to chaos and Hopf bifurcations from fixed points to period- $n$ orbits, $n>2$, or to quasiperiodic orbits are observed. Albeit it is difficult to control the 2-module dynamics (there 
are 6 control parameters involved), it becomes clear, that the coupling of two excitatory or inhibitory units has dynamical effects quite different from those resulting from couplings between excitatory and inhibitory units.

\section{Acknowledgements}

The author would like to thank E. Nelle for stimulating discussions. 


\section{References}

[1] J. J. Hopfield, Neural networks and physical systems with emergent collective computational abilities, Proc. Natl. Acad. Sci. USA, 79, 2554-2558, 1982.

[2] D.E. Rumelhart, G.E. Hinton, R.J. Williams: 'Learning internal representation by error propagation', in: D.E. Rumelhart, J.L. McClelland (eds.), Parallel Distributed Processing: Explorations in the Microstructures of Cognition, Vol. 1, MIT Press, Cambridge M.A., 1986, pp. 318-362.

[3] M. A. Cohen, S. Grossberg, Absolute stability of global pattern formation and parallel memory storage by competitive neural networks, IEEE Transactions on Systems, Man, and Cybernetics SMC-13, 815-826, 1983.

[4] M. W. Hirsch, Convergent activation dynamics in continuous time networks, Neural Networks 2, 331-350, 1989,

M. W. Hirsch, Network dynamics: principles and problems, in: F. Pasemann, H.-D. Doebner (eds.): Neurodynamics, World Scientific, Singapore, 1991, pp. 3-29.

[5] J. A. Anderson, E. Rosenfeld, (eds.) Neurocomputing, MIT Press, Cambridge M.A., 1989.

[6] J. A. Anderson, A. Pellionisz, E. Rosenfeld, (eds.) Neurocomputing 2, MIT Press, Cambridge M.A., 1990.

[7] A. Selverston, P. Mazzoni, "Flexibility of computational units in invertebrate CPGs", in The Computing Neuron, eds. Durbin R. and Miall C. (AddisonWesley, Workingham), pp. 205-228, 1989.

[8] S. L. Bressler, W. J. Freeman, Frequency analysis of olfactory system EEG in cat, rabbit and rat, Electroenceph. clin. Neurophysiol., 50, 19-24, 1980.

[9] C. A. Skarda, W. J. Freeman, How brains make chaos in order to make sense of the world, Behav. Brain Sci., 10, 161-195, 1987.

[10] C. M. Gray, P. König, A. K. Engel, W. Singer, Oscillatory responses in cat visual cortex exhibit inter-columnar synchronization which reflects global stimulus properties, Nature, 338, 334-337, 1989.

[11] A. K. Engel, A. K. Kreiter, P. König and W. Singer, Synchronization of oscillatory neural responses between striate and extrastriate visual cortical areas of the cat, Proc. Natl. Acad. Sci. USA, 88, 6048-6052, 1991.

[12] R. Eckhorn, R. Bauer, W. Jordan, M. Brosch, W. Kruse, M. Munk, H. J. Reitboeck, Coherent oscillations: A mechanism of feature linking in the visual cortex?, Biol. Cybern., 60, 121-130, 1988. 
[13] B. Baird, Nonlinear dynamics of pattern formation and pattern recognition in rabbit olfactory bulb, Physica, 22D, 150-175, 1986.

[14] Z. Li, J. J. Hopfield, Modeling the olfactory bulb and its neural oscillatory processings, Biol. Cybern., 61, 379-392, 1989.

[15] P. König, T. B. Schillen, Stimulus-dependent assembly formation of oscillatory responses: I. Synchronization, Neural Computation, 3, 155-166, 1991.

[16] T. B. Schillen, P. König: "Stimulus-dependent assembly formation of oscillatory responses: II. Desynchronization", Neural Computation, 3, 167-178, 1991.

[17] P. König, B. Janosch and T. B. Schillen, Stimulus-dependent assembly formation of oscillatory responses: III. Learning, Neural Computation, to appear, 1992.

[18] A. Atiya, P. Baldi, Oscillations and synchronizations in neural networks: An exploration of the labeling hypothesis, International Journal of Neural Systems, 1, 103-124, 1989.

[19] D. M. Kammen, P. J. Holmes and C. Koch, Cortical architecture and oscillations in neural networks: Feedback versus local coupling, in: R. M. J. Cotterill (ed.), Models of Brain Functions, Cambridge University Press, Cambridge, 1989.

[20] O. Sporns, J. A. Gally, G. N. Reeke, and G. M. Edelman, Modeling perceptual grouping and figure-ground segregation by means of active reentrant connections, Proc. Natl. Acad. Sci. USA, 86, 7265-7269, 1989.

[21] H. Sompolinsky, D. Golomb, and D. Kleinfeld, Cooperative dynamics in visual processing, Physical Review A, 43, 6990-7011, 1991.

[22] H. G. Schuster (ed.), Nonlinear Dynamics and Neuronal Networks, VCH, Weinheim, 1992.

[23] S. Renals, R. Rohwer, A study of network dynamics, Journal of Statistical Physics, 58, 825-848, 1990.

[24] M. P. Paulus, S. F. Gass and A. J. Mandell, A realistic, minimal "middle layer" for neural networks, Physica, D40, 135-155, 1989.

[25] E. K. Blum, X. Wang, Stability of fixed points and periodic orbits and bifurcations in analog neural networks, Neural Networks, 5, 577-587, 1992.

[26] S. Grossberg, Contour enhancement, short term memory, and constancies in reverberating neural networks, Studies in Applied Mathematics 52, 217-257, 1973. 
[27] J. J. Hopfield, Neurons with graded response have collective computational properties like those of two-state neurons, Proc. Natl. Acad. Sci. USA, 81, 3088-3092, 1984.

[28] R. Thom, Structural Stability and Morphogenesis, Benjamin, Reading, 1975.

[29] E. C. Zeeman, Catastrophe Theory - Selected Papers 1972-1977, AddisonWesley, Reading, 1977.

[30] T. Poston, I. Stewart, Catastrophe Theory and its Applications, Pitman, London, 1978.

[31] R. Gilmore, Catastrophe Theory for Scientists and Engineers, John Wiley, New York, 1981.

[32] S. Wiggins, Introduction to Applied Nonlinear Dynamical Systems and Chaos, Texts in Applied Mathematics 2, Springer Verlag, New York, 1990.

[33] B. G. Cragg, H. N. V. Temperley, Brain, 78, 304, 1955.

[34] E. Harth, T. J. Csermely, B. Beek, R. D. Lindsay, Brain functions and neural dynamics, J. Theoret. Biol., 26, 93-120, 1970.

[35] H. R. Wilson, J. D. Cowan, Excitatory and inhibitory interactions in localized populations of model neurons, Biophysical Journal, 12, 1-24, 1972.

[36] U. an der Heiden, Analysis of Neural Networks, Lecture Notes in Biomathematics 35, Springer Verlag, Berlin, 1980.

U. an der Heiden, Neural networks: Flexible modelling, mathematical analysis, and applications, in: F. Pasemann, H.-D. Doebner (eds.): Neurodynamics, World Scientific, Singapore, 1991, pp. 49-95.

[37] E. C. Zeeman, Differential equations for heartbeat and nerve impulse, in: C. H. Waddington (ed.): Towards a Theoretical Biology 4, Edinburgh University Press, 1972, pp. 8-67. Reprinted in: E. C. Zeeman, Catastrophe Theory Selected Papers 1972-197\%, Addison-Wesley, Reading, 1977.

[38] A. L. Hodgkin, A. F. Huxley, A quantitative description of membrane current and its application to conduction and excitation in nerve, J. Physiol., 117, 500-544, 1952. 